\title{
A Comprehensive Approach to Reliability Optimization of Power Supply to Consumers in Power Interconnection with Weak Tie Lines
}

\author{
Galina Fedotova* \\ Melentiev Energy Systems Institute of Siberian Branch of the Russian Academy of Sciences, 664033 Irkutsk, Russia
}

\begin{abstract}
A new approach is proposed to ensure the optimum level of power supply reliability of the consumers in a multi-zone power interconnection with limited transfer capabilities of tie lines between the zones at the annual planning of the energy systems operation in a market environment. It is based on a stepby-step solution two problems: of distribution of the total reserve of generating capacity in the power interconnection between the zones, taking into account network restrictions, within the zones - between the operational and maintenance components, optimal for the criterion of reliability and planning maintenance of generating equipment optimal for economic criterion. This approach makes it possible to use for reliability the main means available in the conditions of functioning of electric power systems (EPS) - reserves of generating capacity and planned maintenance of the main equipment. At the same time, reliability optimization is carried out taking into account the interests of different subjects of relations at the management of the power interconnection (System operator, generating companies and consumers).
\end{abstract}

\section{Introduction}

The idea to comprehensively solve the problems of the total generating capacity reserve distribution in power interconnection with limited transfer capabilities of intersystem tie lines and planning maintenance of the main equipment of the EPS to ensure the reliability of power supply to consumers in the context of centralized control of the electric power industry belongs to Academician Yu.N. Rudenko [1, 2]. The author of this article has previously developed: methodology, mathematical models and software and computer complex RESERVE for solving these problems, in relation to those conditions $[3,4]$. The problem of power supply reliability remains relevant for the modern restructured energy systems. Research in this area continues and this paper presents a new approach to solving these problems for the case of annual operation planning of electric power systems in a market environment.

The reliability principles used in this approach are consistent with the Federal Law "On Electric Power Industry" [5]. The law states, that: "the reliability function becomes a service (commodity). All the main subjects of the electricity market (generation, networks, sales, consumers) are involved in its purchase and sale operations. At the same time it is necessary to distinguish services on system reliability (balancing of power, reservation, anti-accident actions) and directly power grid reliability of power supply of consumers. All consumers pay for system reliability in the tariff, and consumers who have declared their category of reliability pay for electric network reliability".
Consequently, today the process of ensuring the electric power system reliability should be based on the distribution of responsibility for reliability among all the stakeholders in the electric power industry. Coordination of this responsibility should be based on a combination of government regulation and market principles, including economic, legal and institutional mechanisms. All stakeholders in their areas of responsibility ensure system reliability in EPS, with the main coordinating role assigned to the System operator (SO). The task of the SO is to create generating capacity reserves in the stage of planning the EPS expansion and provide such operation and control conditions under which electrical energy production, transmission, distribution, and consumption, ensuring the required reliability level, are carried out through technological interaction between generating plants, electricity networks and electrical installations of consumers. The reliability of the power supply is determined by the level of system reliability and reliability of power supply. Responsibility for the reliability of power supply to specific consumers is borne by the power supply company (PSC) supplying electrical energy to consumers in a demanded amount following the load curve and power supply contracts provided the consumer fulfills all technical and financial obligations [6].

The division of ownership and the introduction of market mechanisms in the process of managing the electric power industry of Russia led to the emergence of independent stakeholders in the industry that have different interests. The System operator is interested in providing the required reliability, generating companies 
(GC) seek to obtain the maximum profit from the operation of equipment owned by them, and the electrical energy consumers require reliable electricity supply and minimum electricity rates. The proposed approach ensures the optimum level of power supply reliability under dissimilar interests of different stakeholders in the EPS.

\section{Initial positions}

Power interconnection is considered as a complex engineering facility consisting of a set of interconnected areas (concentrated power systems), the transmission lines between which are grouped into intersystem ties. Each area is represented by generation, load and adjacent intersystem ties. Generation in each area is given by the sum of the available capacity of generating units. The availability of generation to cover the load at each time instant is determined by the sum of working capacities of the units, which differs from the available capacity undergoing planned maintenance. The value of the available capacity of each unit is considered to be known for all intervals of the planning horizon and with no specially planned measures remains unchanged during this period. The load in each area is set by the predicted values of the maxima and is considered to be constant at each interval of the planning horizon.

The planning horizon is the coming year, which is divided into discrete time intervals (a month, a week, a day). Ensuring the reliability at this level implies the rational use of the total capacity reserve available in the power interconnection considering the function of its components (operating and maintenance) under the actual situation in power systems and their mutual assistance. The operating reserve should supply load to each area with the required degree of reliability, whereas the task of the maintenance reserve is to replace the capacities to be removed from service for planned maintenance and reconstruction. The optimal maintenance planning is to provide the availability of equipment to cover the load at any time in each area at a rational value of the operating capacity reserve.

In view of the above, in a staged mutually agreed manner solution to the problems of the full capacity reserve distribution between the areas of the power interconnection with weak tie lines and between operating and maintenance components within the areas, as well as the problem of maintenance planning of generating equipment it is proposed.

\section{Statement of the capacity reserve distribution problem}

Stage 1. The problem of generating capacity reserve distribution is solved for the entire power interconnection by the criterion of the maximum possible reliability of power supply to consumers. The level of reliability is estimated using an indicator of relative satisfaction of the consumer demand for electricity. The selected indicator has a simple physical sense, it is quite sensitive to various disturbances leading to a decrease in reliability (emergency power reduction, accidental increase in load, maintenance of equipment) and to measures that improve the reliability (commissioning of new equipment, increase in the transfer capability of ties, redundancy) [1].

The power interconnection is represented by a design scheme, in which the nodes are the areas of free power flow or lumped power systems, and the connections are intersystem tie lines. Each node of the design scheme includes points where electricity suppliers and consumers are connected to the electrical network.

In the specified initial conditions, the maximum possible reliability of power supply to consumers is to be provided at each node of the design scheme at all the planning horizon intervals through the rational use of the total generating capacity reserve available in the power interconnection. The total reserve is optimally distributed between the nodes of the design scheme and at the nodes, the reserve is distributed between its operating and maintenance components, considering power balances at the nodes and transfer capabilities of intersystem links. The operating component of the reserve ensures the highest possible reliability of the power supply to consumers, while the maintenance component provides for the annual volume of planned maintenances of generating equipment. The strategic capacity reserve is taken into account according to the standard.

\subsection{A mathematical model}

Find the maximum of a function of power supply reliability at nodes by time interval

$$
\begin{gathered}
F\left[_{\mu j} \operatorname{ICAP}_{\mu j}, L O A D_{\mu j}, R O_{\mu j}, P L_{l j}\right] \rightarrow \max , \\
\mu=\overline{1, M} ; j=\overline{1, G}, l=\overline{1, L}
\end{gathered}
$$

subject to power balance constraints on the nodes and its components:

$$
\begin{gathered}
I C A P_{\mu j}-L O A D_{\mu j}-R O_{\mu j}-R P_{\mu j}-R S_{\mu j} \pm \sum_{l} P L_{l j}=0 ;(2) \\
\underline{R O}_{\mu} \leq R O \overline{\mu j}_{\mu j} \leq \overline{R O} \\
\sum_{j=1}^{G} R P_{\mu j} \Delta T_{j}=V_{\mu} ; \\
\underline{P L}_{l} \leq P L_{l j} \leq \overline{P L}_{l}
\end{gathered}
$$

where: $M$ - the number of nodes in a design scheme; $G$ - the number of discrete time intervals in the planning horizon; $L, L \mu^{-}$the number of ties between the nodes in the design scheme and ties adjacent to the $\mu$-th node; ICAP $\mu_{j}$ - available capacity in the $\mu$-th node at the $j$ 
-th time interval; $L O A D \mu_{j}-$ load of the $\mu$-th node at the $j$-th time interval; $R O{ }_{\mu j}, \underline{R O}_{\mu}, \overline{R O} \mu^{- \text {calculated }}$ value of operating capacity reserve in the $\mu$-th node at the $j$-th time interval and its maximum permissible value in the $\mu$-th node; $R P \mu_{j}{ }^{-}$calculated value of the maintenance capacity reserve in the $\mu$-th node at the $j$ th time interval; $\Delta T_{j}$ - duration of the $j$-th discrete time interval in the planning horizon; $V_{\mu}$ - annual volume of planned maintenance of generating equipment in the $\mu$ th node; $R S \mu_{j}^{-}$a set (standard) value of strategic capacity reserve of the $\mu$-th node at the $j$-th time interval; $P L_{l j}, \underline{P L}_{l}, \overline{P L}_{l}-$ calculated power flow by the $l$-th tie line at the $j$-th time interval and its transfer capabilities.

The indicator of relative satisfaction of the consumer demand for electricity at the $\mu$-th node at the $j$-th time interval is determined by the equation:

$$
\pi_{\mu j}=1-M\left(\Delta W_{\mu j}\right) / W_{\mu j}
$$

Consequently, the objective function of the problem is represented by the following expression:

$$
\begin{gathered}
1-M\left(\Delta W_{\mu j}\right) / W_{\mu j} \rightarrow \max , \\
\mu=\overline{1, M} ; j=\overline{1, G},
\end{gathered}
$$

where: $M\left(\Delta W_{\mu j}\right)-$ mathematical expectation of electricity undersupply to the consumer at the $\mu$-th node in the $j$-th time interval; $W_{\mu j}$-predicted volume of electricity consumption at the $\mu$-th node at the $j$-th time interval.

The mathematical expectation of electricity undersupply is determined based on the probabilities of power shortage, which, in the case of the insufficient reserve, can be caused by both an emergency failure of power plant equipment and a sudden increase in load due to random factors. All possible values of the power shortage probabilities are determined by multiplying the probabilities of an emergency power reduction and a load deviation from a predicted value. The value of the projected volume of electricity is calculated taking into account the error of the load forecast.

By solving problem (1) - (7), the following characteristics are determined in the nodes of the design scheme at each interval of the planning horizon: the values of the operating and maintenance components of the total capacity reserve, the power flows over the tie lines that ensure the maximum possible or guaranteed level of power supply reliability. Taking into account the current state of the industry and increased requirements for the reliability of power supply to consumers, the level of guaranteed reliability may prove insufficient for all consumers. As a result, very large consumers may suffer huge losses from power undersupply and its consequences. In such cases, ensuring the required level of reliability of power supply to specific consumers becomes a problem of the power supply companies and a subject matter of market relations $[6,7]$.

The power supply companies (PSC), having received a projection of the guaranteed reliability of power supply for the coming year from the System operator, assess whether it meets the reliability requirements of the consumer served by them. The use of the market for system services and a variety of means and measures allows the PSC to provide the levels of power supply reliability demanded by consumers at additional cost. In this case, consumers satisfied with the guaranteed level of reliability pay for electricity at a base rate. If the required level of reliability is lower than the guaranteed one, consumers can get a discount, in case of its excess - a premium to the tariff, which is determined by the cost of improving reliability [8].

There have always been problems of insufficient and surplus capacity in EPS and solving them requires effective control systems, great efforts, and investment. Today, active consumer of electricity can influence their level of power supply reliability, for the sake of saving and earning, they increasingly more often seek to provide themselves with electricity either completely or in part, they can generate electricity for themselves and even sell it. The emergence of prosumers, i.e. consumers selling surplus electricity they generate to the public network and the development of distributed generation can help to solve the problem of insufficient power to ensure the reliability of power supply to consumers.

\section{Statement of the maintenance planning problem}

Stage 2. The problem of maintenance planning is solved sequentially for all nodes of the design scheme according to the criterion of the maximum profit of generating company given the reliability requirements of consumers. The statements of maintenance planning problems and approaches to solving them are determined by the maintenance strategies used and the operating conditions of the power systems. Given the current context (highly worn equipment, low rates of commissioning new energy capacities, increased power supply reliability requirements, etc.), a combination of strategies for planned preventive maintenance (PPM) and conditionbased maintenance is used. The combination of these maintenance planning strategies makes it possible to ensure the required level of reliability and improve the efficiency of equipment operation, and thus take into account the interests of electricity producers and consumers $[9,10]$. 
Based on the PPM strategy, power plants submit applications for the equipment maintenance to the SO of the regional dispatch control office for the coming year. These applications are agreed with the generating company and transferred to the $\mathrm{SO}$ of the integrated dispatch control center. Based on these applications, a common ranked maintenance list is formed in the each node, given the technical condition of the equipment, reduction in the available capacity of power plants during maintenance and maintenance time. To this end, the rating (RE) of each maintenance at the $\mu$-th node is determined. The level of technical condition of the equipment is assessed using a conventional index $T C=\overline{0,1}$. Its value is determined by the results of technical diagnostics [9]. The lower this index, the more dangerous further operation of the equipment. The rating of maintenance is determined by the formula:

$$
R E_{\mu i}=\operatorname{ICAP}_{c \mu i^{\tau}{ }_{\mu i}\left(1-T C_{\mu i}\right), i=\overline{1, I},},
$$

where: $I_{\mu}$ - the number of maintenance in the list of the $\mu$-th node; $I C A P_{c \mu i}-$ a reduction of the available capacity of the power plant during the $i$-th maintenance in the $\mu$-th node; $T C_{\mu i}$-index of technical condition of the $i$-th unit in the list of the $\mu$-th node; $\tau_{\mu i}-$ duration of the $i$-th maintenance in the $\mu$-th node.

In the list, the planned maintenance are arranged in order of decreasing ratings. Ranking of the maintenance according to this principle makes the long-term maintenance of economical high-power equipment in the poor technical condition of top priority. The planned maintenance of such equipment in optimal time creates favorable conditions for the operation of generating company. Short-term maintenance of low-power equipment can be performed during week-long and daylong load dips without loss of reliability, it does not require large technical and financial resources. Maintenance that continues from the previous year, or the one with fixed dates, is automatically included in the maintenance schedule and does not participate in optimization. For the obtained ranked maintenance list, the maintenance planning problem is solved at each node in the following statement.

For each element of the maintenance list are known: the type of unit, its available capacity and reduction in the power plant output due to the maintenance of this unit, the maintenance duration and conditions. It is necessary to choose the optimal time of maintenance, included in the list, to provide the maximum possible annual profit of generating companies from the operation of the equipment owned by them, given the required level of power supply reliability. The optimization of the maintenance time takes into account the initial conditions, including seasonal constraints; incompatibility of certain maintenance; contracts with maintenance companies; the presence of maintenance reserve.

\subsection{A mathematical model}

Find the maximum profit function of GC of the $\mu$-th node when optimizing an annual schedule of planned maintenance of a specified maintenance list

$$
P_{\mu}\left(A I_{\mu}, A P C_{\mu}, P E N_{\mu}, B_{\mu i}, E_{\mu i}\right) \rightarrow \max
$$

considering:

- seasonal constraints on maintenance time, which for the equipment of steam plants is associated with heat load balance to be provided, and for the equipment of hydropower plants - with the period of flood, when the removal from service of units leads to an additional idle discharge of water,

$$
\begin{gathered}
T_{b \mu i \beta} \leq B_{\mu i} \leq T_{e \mu i \beta}-\tau \mu i \\
i=\overline{1, I} \mu, \beta=\overline{1, n_{i}} \\
B_{\mu i}=E_{\mu i}^{-\tau_{\mu i}}
\end{gathered}
$$

- incompatibility of periods of certain maintenance,

$$
B_{\mu i}>E_{\mu m} \vee E_{\mu i}<B_{\mu m}, \mu m \in I H_{\mu i} ;
$$

- limitations on the capacity to be repaired by maintenance companies in the $\mu$-th node at the $j$-th time interval on the contracts with power plants,

$$
\sum_{k=1}^{K} P M_{\mu k j} \leq P R K_{\mu j}
$$

- limitations on the valume of maintenance capacity reserve in the $\mu$-th node at the $j$-th time interval,

$$
\sum_{k=1}^{K} P M_{\mu k j} \leq R P_{\mu j},
$$

where: $P R F{ }_{\mu}$-annual profit of the GC in the $\mu$-th node; AI $\mu^{- \text {total annual income of the GC from the supply of }}$ electricity and capacity to the market of the $\mu$-th node; APC $\mu^{- \text {total production costs of the GC of the } \mu \text {-th }}$ node; $P E N{ }_{\mu}$ - penalty of the GC of the $\mu$-th node for electricity and capacity undersupply to the market due to untimely maintenance of generating equipment; $I_{\mu}$-the number of maintenance in the ranked maintenance list of 
the $\mu$-th node; $B \mu i, E_{\mu i}$-calculated values of the beginning and end of the $i$ - th maintenance in the $\mu$-th node, respectively; $T \mu b i \beta, T$ нe $i \beta^{- \text {time limits of }}$ seasonal constraints within which the $i$-th maintenance of the $\mu$-th node is allowed; $n i^{-}$the number of intervals during which the $i$-th maintenance in the $\mu$-th node can be performed; $I_{\mu i}{ }^{- \text {a set of maintenance }}$ whose performance time should not coincide with the time of the $i$-th maintenance in the $\mu$-th node; $K_{\mu}^{-}$ the number of power plants in the $\mu$-th node; $P M \mu k j$ - the calculated value of the total generating capacity of power plants in the $\mu$-th node, which is planned for maintenance according to the schedule at the $j$-th time interval; $P R K_{\mu j}-$ capacity to be repaired by a maintenance company, which is stipulated by the contract for maintenance of equipment of the $\mu$-th node at the $j$

-th time interval; $R P_{\mu j}$ - the maintenance capacity reserve of the $\mu$-th node, intended for planned maintenance during the $j$-th time interval without loss of reliability, obtained by solving problem (1) - (7).

Annual profit of the GC of the $\mu$-th node is determined by the difference between the total annual revenue from delivery of electricity and capacity to the market and cumulative production costs, given penalties for electricity and capacity undersupply to the market due to possible unplanned maintenance of generating equipment of power plants of the $\mu$-th node. In these initial conditions, the objective function of the problem is represented by the following expression:

$$
\left.P R F_{\mu}=\left\{A I_{\mu}^{-\left(A P C_{\mu}+P E N\right.} \mu\right)\right\} \rightarrow \max
$$

All components of the objective function directly or indirectly depend on the timing of the generating equipment maintenance, since they include the volumes of electricity and capacity deliveries depending on the composition of the operating equipment of power plants, reducing their capacity during the equipment maintenance and the costs changing over time in a market environment. In this regard, the annual profit of the generating company is also a function of the maintenance timing.

The penalty component is introduced in the objective function to account for violations generating companies contracts for the delivery of electric energy and power to the market because of unplanned maintenance of generating equipment. This component makes it possible to reconcile the interests of the System operator and generating companies with each other and with electricity consumers while optimizing the timing of planned maintenance. What is meant here is the equipment, which, following the standards, should be removed from service for planned maintenance in the coming year, but for some reasons, its maintenance is postponed. If maintenance is carried out within the planned timeframes agreed upon with the System operator, then the contract for the delivery of electric energy and power indicates the periods of decline in the delivery of generating company products due to the removal from service of the equipment of power plants and the contract is not violated. Unplanned maintenance of equipment can reduce the available capacity of the power plant during the maintenance, affect the electricity output and lead to a failure to fulfill the contractual obligations. To eliminate the risk of lower reliability, in the case of the failure of generating company to fulfill the contract for the delivery of electric energy and power to the market, the capacity reserve of other companies is used. However, since the cost of the additionally generated electricity is higher than that of the planned one, the generating company that breached the contract must reimburse additional costs by paying a fine. The proposed approach was tested in a case study, the algorithm for solving problem (9) - (15) is given in [11].

The discrete nature of the maintenance planning problem does not allow the full use of the maintenance reserve, obtained by solving problem (1) - (7), at all intervals of the planning horizon. This makes it, on the one hand, impossible to include of the all the maintenance in the schedule and, on the other hand, leads to a change in the level of reliability. All this requires redistribution of the operating reserve between the nodes, considering the obtained maintenance schedules and the assessment of the power supply reliability level. To this end, problem (1) - (7) is solved again without constraints (4) and the magnitude $R P_{\mu j}$ in the balance equation (2) is an initial one. It is determined by summing up the capacity of the equipment removed from service for maintenance at the nodes at each interval of the planning horizon according to the schedules obtained in step 2 .

\section{Conclusion}

A new approach has been proposed to optimize the reliability of power supply to consumers in a multi-area power interconnection with limited transfer capabilities of tie lines between the areas with a view of annual planning of energy systems operation in a market environment. Are present a methodology mutually agreed solution of the task of distribution of capacity reserves and the planning of maintenance, a meaningful formulation and mathematical models tasks.

Basics of ensuring reliability in the proposed approach comply with the principles adopted by the Federal Law "On the Electric Power Industry". The novelty of the proposed approach lies in the fact that it, following the law "On Electric Power Industry", takes into account the specific features of the restructured EPS operation and 
actual conditions of energy equipment operation and maintenance in a market environment. A mechanism has been proposed to cope with the issues arising between the System operator and generating companies in the process of reliability optimization and to reconcile their interests in a market environment.

Solving problems (1) - (7) and (9) - (15) jointly makes it possible to ensure the optimal level of power supply reliability by using two main means - generating capacity reserves and planned maintenance. The optimal maintenance reserve (in terms of reliability), which is obtained by solving the first problem, is a constraint in the second problem where a maintenance schedule optimal by economic criterion is formed. Violation of this constraint may entail a decrease in the reliability of power supply to the consumers in the node and a penalty of the System operator.

In solving the maintenance planning problem employs an advanced approach, i.e. a combination of two strategies: planned preventive maintenance and condition-based maintenance. The planned maintenance schedule is optimized according to the criterion of maximum profit of generating companies, which can be provided either by maximizing the total annual revenue, or by minimizing the total production costs given the penalty for breach of the contract for electric energy and power delivery to the market, or by both. A component of a penalty imposed on generating company for a breach of the contract for the delivery of electric energy and power to the market due to unplanned maintenance of generating equipment is introduced in the objective function. This makes it possible to coordinate the interests of the System operator, the generating companies and the electricity consumer when determining the time of planned maintenance.

The research was carried out under State Assignment, Project 17.5.3 (reg. no. AAAA-A17-117030310450-3) of the Fundamental Research of Siberian Branch of the Russian Academy of Sciences.

\section{References}

1. Yu.N. Rudenko, M.B. Cheltsov, Reliability and redundancy in electric power systems. Methods of research (Novosibirsk, Nauka, Siberian Branch, 1974)

2. Yu.N. Rudenko, I.A. Ushakov, Reliability of energy systems (Novosibirsk, Nauka, 1989)

3. G.A. Fedotova, Methods of rational use of generating capacity reserves in an energy interconnection, Proc. of the RAS. Power Engineerin, 3, 108-120 (2013)

4. G.A. Fedotova, Maintenance planning for the main equipment of powerplants and transmission lines of intersystem tie lines, Reliability of energy systems and their equipment. Reference book, V 2, 97-110 (Msc., Energoatomizdat, 2000)

5. Federal Law of the RF "On Electric Power Industry" No. 35 (March 26, 2003)

6. The reliability concept in the electric power industry (Ministry of Energy of the RF, 2011)

7. N.I. Voropai, Reliability of power supply systems. (Novosibirsk, Nauka, 2015)

8. V.I.Edelman, The market for electric energy and power in Russia: what it should be (Msc., Energoatomizdat, 2000)

9. A.N. Nazarychev, Methods and Models for Optimization of Condition-Based Maintenance of Electrical Equipment of Energy Facilities (Ivanovo: ISU, 2002)

10. A.V. Andryushin, E.Yu. Shnyrov, Management of Maintenance Service of Energy Equipment under New Conditions, Methodological problems in reliability study of large energy systems, Iss.54, Book 2, 145-156 (Irkutsk, ESI SB RAS, 2005)

11. N.I. Voropai, G.A. Fedotova, Planning Maintenance of Power Generating Equipment in Market Environment with Regard for Reliability, Automation and Remote Control, T.71, 7, 1442-1446 (2010) DOI: $10.1134 / \mathrm{S} 0005117910070180$ 